\title{
Virulence of Cryptococcus neoformans serotypes A, B, C and $D$ for four mouse strains
}

\author{
E. A. O. IROKANULO and C. O. AKUESHI* \\ Epidemiology Research Division, National Veterinary Research Institute, Vom and *Botany Department, \\ University of Jos, Nigeria
}

\begin{abstract}
Summary. The relative virulence of Cryptococcus neoformans serotypes A, B, C and D in four mouse strains was assessed by measuring their migration from the foot-pad of the animals to the spleen, lungs and brain in 6-week-old DBA/2, BALB/c, A/J and a hybrid mouse strain by re-isolating yeasts from the internal organs. Comparable doses of each $C$. neoformans serotype were inoculated into the foot-pads of the mice. C. neoformans var neoformans strains A68, D52, A-(IN) and D-(IN) were more virulent than $C$. neoformans var gatti strains $\mathrm{B} 112$ and $\mathrm{C} 18$. However, the differences in the relative virulence of the var neoformans and the var gatti serotypes for the mouse strains were not significant $(p>0.05)$. Re-isolation of yeasts from mice showed that the BALB/c mice, in particular, and the DBA/2 mice were more susceptible to disseminated $C$. neoformans infection. The virulence of $C$. neoformans serotypes through foot-pad inoculation of mice was established.
\end{abstract}

\section{Introduction}

Cryptococcosis is commonly acquired by inhalation and the capsule of Cryptococcus neoformans has been identified as a virulence factor. ${ }^{1,2}$ Other possible routes of infection have been assessed and one such route is the alimentary tract in immunodeficient hosts. ${ }^{3}$ Mucosal surfaces can be colonised by the capsulate strains of $C$. neoformans, from where they may cause systemic cryptococcosis. ${ }^{3}$ Asymptomatic infection in man and animals is common; the clinical manifestations become apparent in conditions of stress and immunosuppression. ${ }^{4}$ Four serotypes of this yeast are known-A, B, C and D. All can cause systemic disease. Most infections in immunodeficient patients with AIDS are believed to be caused by $C$. neoformans var neoformans (serotypes A, D). ${ }^{5}$ Dissemination of infection becomes enhanced in cases associated with hypogammaglobulinaemia ${ }^{6}$

Previous attempts to induce resistance in mice experimentally through different routes of inoculation with live and killed, virulent and sometimes avirulent strains, have been unsatisfactory. ${ }^{7,8}$ On the other hand, survival in mice may be enhanced by immunisation with anti- $C$. neoformans capsular polysaccharide or monoclonal antibodies. ${ }^{9,10}$ Furthermore, it is not known whether cutaneous inoculation does result in immunisation. Cryptococcal lesions produced in the lung, as a result of host defence mechanisms against invading cryptococci, are determined by both the immunological competence of the host and the virulence of the invading yeast. ${ }^{11}$ Cryptococcosis may be fatal, depending on the inoculum size, when the route of inoculation is intravenous or intraperitoneal. ${ }^{12,13}$ Although skin cryptococcomas may arise, as demonstrated in guinea-pigs inoculated intraperitoneally,${ }^{12}$ there are doubts as to whether systemic infection occurs when viable $C$. neoformans are implanted cutaneously or subcutaneously, in spite of the partial success reported earlier. ${ }^{8.14}$ The present study examined the susceptibility of four mouse strains to the relative virulence of Cryptococcus neoformans serotypes A, B, C and D inoculated into the footpad.

\section{Materials and methods}

\section{Experimental animals}

Two hundred and twenty-four 6-week-old mice belonging to four strains-DBA/2, BALB/c, A/J and a hybrid (crossed from DBA/2 and CBA) - were used. All the mice were inbred at the National Institute for Trypanosomiasis Research, Vom, Nigeria.

\section{Organisms}

Seven strains of $C$. neoformans were used; they included all four serotypes designated $C$. neoformans var neoformans (strains A68, A551, D52) and $C$.

Received 4 Oct. 1994; revised version accepted 14 March 1995 
neoformans var gatti (B112 and $\mathrm{C} 18)$; these were provided by Dr J. E. Bennett, NIH, Bethesda, MD, USA, and Dr R. Ikeda, Department of Microbiology, Meiji College of Pharmacy, Tokyo, Japan. Two further strains, $C$. neoformans var neoformans of serotypes designated as A(IN) and D(IN) were isolated from bird droppings in Nigeria. All the $C$. neoformans strains except $C$. neoformans var neoformans A551 were capsulated.

\section{Preparation of yeast inocula}

Viable yeast cells were harvested from 48-h Sabouraud agar cultures into sterile phosphatebuffered saline (PBS) and the concentrations were adjusted to $10^{7}$ organisms $/ 0.02 \mathrm{ml}$. The concentration of the yeast in the inoculum was confirmed by viable counts in replicate pour-plates.

\section{Animal inoculation}

Eight mice from each strain were inoculated with each $C$. neoformans serotype strain. Each group of eight mice contained an equal number of males and females. The yeasts were injected into the left hind foot-pad of the mice. Fourteen mice from each strain were used as controls and were inoculated with $0.02 \mathrm{ml}$ of sterile PBS.

\section{Re-isolation of yeast}

The experiments were terminated 2 weeks after footpad inoculation. Feed was withheld from the mice for $18 \mathrm{~h}$ before the animals were killed; $\mathrm{CO}_{2}$ gas was piped into polythene bags containing the mice. At death, they were examined for skin infection, rough fur coats and for foot-pad lesions. Tissues from the site of inoculation, spleen, lung and brain were removed and examined for lesions and a qualitative examination of each of the organs for the presence of the various $C$. neoformans serotype strains was made by culture.

\section{Culture}

Skin covering the foot-pads was removed and the dermis was smeared on the surface of Sabouraud and malt-extract agar. Spleen, lung and brain tissues minced and homogenised in PBS were filtered through clean sterile muslin and the filtrate was centrifuged at $2000 \mathrm{~g}$ for $5 \mathrm{~min}$. The pellets were used to inoculate Sabouraud and malt-extract agar containing chloramphenicol $0.05 \mathrm{mg} / \mathrm{ml}$. Duplicate cultures were incubated at $25^{\circ} \mathrm{C}$ and $37^{\circ} \mathrm{C}$ for $24-120 \mathrm{~h}$. Isolates were re-identified by standard procedures. ${ }^{15,16}$ All the data generated from this investigation were analysed statistically by the analysis of variance (ANOVA) at the $5 \%$ level.

\section{Results}

At post-mortem examination, lesions characteristic of $C$. neoformans infection in the foot-pad, spleen and lung were observed in some of the infected mice but

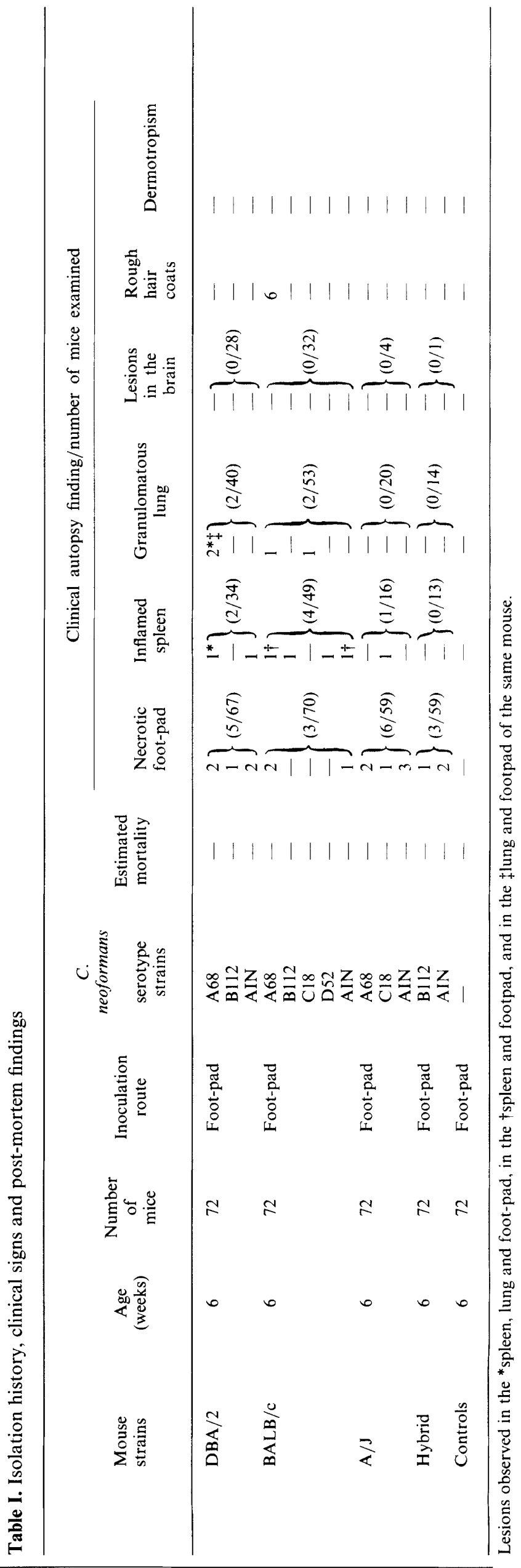


not in the controls, Necrotic foot-pads, inflamed spleens and granulomatous lungs were seen in 23 of the mice inoculated with three $C$. neoformans var neoformans serotype strains A68, D52 and AIN, and two C. neoformans var gatti serotype strains B112 and $\mathrm{C} 18$. Of the C. neoformans serotype strains used, strain A68 caused more lesions than the other strains in the foot-pads, spleens and lungs of the DBA/2, BALB/c and $\mathrm{A} / \mathrm{J}$ mice. The local isolate, $C$. neoformans var neoformans $\mathrm{A}(\mathrm{IN})$ caused lesions in all four mouse strains but with less involvement of the lungs. Only one BALB/c mouse inoculated with C. neoformans var neoformans D52 had an inflamed spleen. Lesions were caused in the BALB/c mice by $C$. neoformans serotype strains $\mathrm{A} 68, \mathrm{~B} 112, \mathrm{C} 18 \mathrm{D} 52$ and $\mathrm{A}(\mathrm{IN}))$, and in $\mathrm{DBA} / 2$ and $\mathrm{A} / \mathrm{J}$ mice by $C$. neoformans serotype strain A68, B112, A(IN), and A68, C18 and A(IN), respectively. Lesions in the hybrid mice were restricted to the foot-pads only and were caused by $C$. neoformans var neoformans $\mathrm{A}(\mathrm{IN})$ and $C$. neoformans var gatti B112. Six of the BALB/c mice inoculated with $C$. neoformans var neoformans A68 showed rough hair coats. Brain tissues from none of the mouse strains manifested any observable lesions and there were no signs of dermotropism in any of the mice. Two deaths were recorded among the BALB/c mice, one each on the fourth and ninth day respectively after inoculation. The results are summarised in table $I$.

\section{Re-isolation of C. neoformans serotype strains}

A positive virulence test in this investigation was based on the re-isolation of the $C$. neoformans strains from one or more tissues other than the foot-pad. Migration of the yeasts from the foot-pad to the spleen, lungs and brain occurred in $108(48.2 \%)$ of the mice. Culture showed that migration was more common in the DBA $/ 2$ and BALB/c mice than in the $\mathrm{A} / \mathrm{J}$ and hybrid strains. However, there was a difference in the number of positive cultures recorded from the tissues of the DBA/2 and BALB/c mice for the different $C$. neoformans strains; more isolates were made from tissues of the BALB/c mice. However, the results did not differ statistically $(p>0.05)$ except for results obtained with mouse strains $\mathrm{DBA} / 2$ and $\mathrm{BALB} / \mathrm{c}$ inoculated with $C$. neoformans var neoformans A551. Significant differences in virulence for the mice, as recorded from the number of isolates, were found between the DBA/2 and $\mathrm{A} / \mathrm{J}$ mice and between DBA $/ 2$ and hybrid mice $(p<0.05)$. Similar results were obtained when the virulence of the various C. neoformans serotypes in the BALB/c mice was compared with that in the $\mathbf{A} / \mathbf{J}$ and hybrid mice. Only one hybrid mouse yielded a positive brain culture of C. neoformans var neoformans A68, whereas four isolates-one of strain A68 and three of strain $\mathrm{A}(\mathrm{IN})$-were made from the brains of the $\mathrm{A} / \mathrm{J}$ mice (table II).

The results showed that $C$. neoformans var neoformans strains $\mathrm{A} 68, \mathrm{~A}(\mathrm{IN})$ and $\mathrm{D} 52$ were more invasive than $C$. neoformans var gatti strains B112, and C18. Overall, $C$. neoformans var neoformans strain A68 was more virulent, being re-isolated to a greater extent from the spleens, lungs and brains of the mice. With the exception of the foot-pads, the lungs remained the most frequently infected organ whereas re-isolation of the yeasts was least from the brain: 99 isolations of the seven yeasts were made from lung specimens, 85 from spleen and 64 from brain. Positive brain cultures were obtained in 13 instances with $C$. neoformans var neoformans $\mathrm{A} 68$, on 11 occasions with C. neoformans var neoformans D52 and A(IN), and on 10 occasions each with $C$. neoformans var gatti $\mathrm{C} 18$ and $C$. neoformans var neoformans $\mathrm{D}(\mathrm{IN})$. Nine isolates of strain B112 were made from brain specimens but no isolate of $C$. neoformans var neoformans A551.

\section{Discussion}

Common symptoms in mice inoculated or infected with $C$. neoformans include listlessness, rough hair coat, hydrocephalus-like bulging of the cranium and,

Table II. Organs from which $C$. neoformans serotype strains were recovered according to mouse strains*

\begin{tabular}{|c|c|c|c|c|c|c|c|c|c|c|c|c|c|c|c|c|}
\hline \multirow{3}{*}{$\begin{array}{l}\text { C. neoformans } \\
\text { serotype strain }\end{array}$} & \multicolumn{16}{|c|}{ Number of mice from which $C$. neoformans was recovered } \\
\hline & \multicolumn{4}{|c|}{$\mathrm{DBA} / 2$} & \multicolumn{4}{|c|}{$\mathrm{BALB} / \mathrm{c}$} & \multicolumn{4}{|c|}{$\mathrm{A} / \mathrm{J}$} & \multicolumn{4}{|c|}{ Hybrid } \\
\hline & $\mathrm{Fp}$ & $\mathrm{Sp}$ & Ln & $\mathrm{Bn}$ & Fp & $\mathrm{Sp}$ & $\operatorname{Ln}$ & $\mathrm{Bn}$ & $\mathrm{Fp}$ & $\mathrm{Sp}$ & $\operatorname{Ln}$ & $\mathrm{Bn}$ & Fp & $\mathrm{Sp}$ & $\operatorname{Ln}$ & $\mathrm{Bn}$ \\
\hline A68 & 8 & 7 & 6 & 6 & 8 & 7 & 8 & 5 & 5 & 2 & 3 & 1 & 6 & 1 & 2 & 1 \\
\hline A551 & 8 & 0 & 0 & 0 & 7 & 2 & 1 & 0 & 8 & 0 & 2 & 0 & 6 & 2 & 0 & 0 \\
\hline B 112 & 8 & 3 & 5 & 3 & 8 & 4 & 7 & 6 & 8 & 1 & 3 & 0 & 7 & $\overline{1}$ & 0 & 0 \\
\hline C18 & 8 & 5 & 7 & 6 & 8 & 3 & 5 & 4 & 8 & 0 & 0 & 0 & 7 & 0 & 2 & 0 \\
\hline D52 & 8 & 6 & 7 & 6 & 8 & 6 & 6 & 5 & 7 & 2 & 3 & 0 & 8 & 2 & 2 & 0 \\
\hline$A(I N)$ & 8 & 3 & 5 & 3 & 7 & 7 & 5 & 5 & 7 & 6 & 5 & 3 & 7 & 1 & I & 0 \\
\hline $\mathrm{D}(\mathrm{IN})$ & 7 & 6 & 6 & 4 & 8 & 7 & 7 & 6 & 4 & 1 & 0 & 0 & 6 & 0 & 1 & 0 \\
\hline Controls & 0 & 0 & 0 & 0 & 0 & 0 & 0 & 0 & 0 & 0 & 0 & 0 & 0 & 0 & 0 & 0 \\
\hline Total & 55 & 30 & 36 & 28 & 54 & 36 & 39 & 31 & 47 & 12 & 16 & 4 & 47 & 7 & 8 & 1 \\
\hline
\end{tabular}

Fp, foot-pad; Sp, spleen; Ln, lung; Bn, brain.

*Eight mice of each strain were inoculated with each serotype. 
in terminal conditions, marked signs of central nervous system disturbance. In the present study, rough hair coats were seen in six BALB/c mice inoculated with $C$. neoformans var neoformans strain A68.

C. neoformans kills 6-week-old Swiss white mice inoculated intracerebrally with a $0 \cdot 02-0 \cdot 03-\mathrm{ml}$ inoculum between 4 and 14 days. ${ }^{17}$ An inoculum of $10^{6}-10^{7}$ cells/mouse given intravenously or intraperitoneally also yields an LD50 of $1.4 \times 10^{3}$ cells/mouse with an atypical $\mathrm{H} 140$ strain. $^{13}$ Findings in this study have differed from an earlier report on subcutaneous implantation of viable $C$. neoformans in mice which resulted in the death of two animals 80 days after inoculation. ${ }^{8}$ Two BALB/c mice died between the fourth and ninth day after foot-pad inoculation. However, these deaths could not be traced to infection with $C$. neoformans at autopsy and from culture of the internal organs. It may have been possible to record some deaths due to cryptococcosis during this investigation if the duration of infection had been allowed to proceed beyond 2 weeks. The route of inoculation may contribute to the speed of spread of the inoculated $C$. neoformans; the cerebral, intravenous, intraperitoneal and subcutaneous routes have all resulted in lethal infections.

This study concentrated on demonstrating the relative susceptibility of four mouse strains to the spread of $C$. neoformans from the foot-pad to the spleen, lung and brain within 2 weeks. Therefore, the number of yeast cells recovered from the cultured organs were not estimated by viable counts, but colony counts for each positive culture ranged from 22 to $>150$, with the brain cultures always yielding lower numbers whilst the foot-pads yielded higher counts.

Cryptococcosis has been elicited experimentally through different routes with the mouse model. Such routes include the intravenous, subcutaneous and intraperitoneal routes, although cutaneous cryptococcosis may resolve spontaneously. ${ }^{8,13,18,19}$ No sick animals were observed throughout the 2-week period of this study. Again, this time period may be responsible for the lack of apparent physical clinical signs: isolation from the brains of the mice suggests that if a longer incubation period was allowed, some clinical signs associated with CNS disease could result. No attempts were made to demonstrate antibodies in the sera of mice used, but the yeasts were demonstrated by culture in $48.2 \%$ of the animals' internal organs. This suggests that migration from the site of inoculation to other organs had occurred. This is comparable to reports in which mice were inoculated intravenously through the tail with $C$. neoformans. ${ }^{8}$

\section{References}

1. Kozel TR. Non-encapsulated variant of Cryptococcus neoformans. II Surface receptors for cryptococcal polysaccharide and their role in inhibition of phagocytosis by polysaccharide. Infect Immun 1977; 16: 99-106.
The mice used in this investigation can be regarded as immunologically competent considering the number that manifested lesions in the lungs. ${ }^{11}$ Therefore, the six BALB/c mice inoculated with $C$. neoformans var neoformans A68 that showed rough hair coats, a common symptom in mice infected with $C$. neoformans, further suggests that there was some degree of greater virulence of the yeasts for these mice.

Not all the tissues examined at autopsy revealed recognisable lesions. As expected, lesions were more evident in the foot-pads (table I). Seibold and colleagues have reported that no gross lesions were seen in the brain of an infected dog but the clinical signs of meningitis and encephalitis were seen. ${ }^{20}$ Although it may not be adequate to compare clinical signs due to cryptococcosis in a dog's brain with that of mice, findings in this study suggest that the visual recognition of lesions arising from cryptococcal infection in the brains of mice is difficult. This apparent lack of lesions due to $C$. neoformans in the brain may be attributed to the relatively low numbers of viable $C$. neoformans cells recovered on culture.

Virulence can be defined as an organism's capacity to spread beyond the drainage lymph node and so cause disease. The $C$. neoformans serotypes used in this study can be considered relatively virulent, having been re-isolated from the spleens, lungs and brains of the inoculated mice. Apart from $C$. neoformans var neoformans strain A551, whose capsule size before inoculation was small, there were no major differences in the degree of invasiveness of the $C$. neoformans serotypes A, B, C and D used. Migration of the yeasts from the foot-pad through to the brain was greater in the BALB/c and DBA/2 mice and this was due more to the C. neoformans var neoformans serotypes (strains A68, AIN, D52 and DIN). The ability of $C$. neoformans var neoformans strains A68, AIN and D52 to invade the mouse tissues more frequently than the var gatti strains confirms the epidemiological report that the serotype A, in particular, may be a more common cause of clinical disease. ${ }^{21}$ The other serotypes (strains B112 and C18) also showed considerable virulence in the same mouse strains (BALB/c and $\mathrm{DBA} / 2)$. Therefore, the difference in the relative virulence of the $C$. neoformans serotypes for the different mouse strains suggests that $B A L B / c$ mice, in particular, and the DBA/ 2 mice are more susceptible to $C$. neoformans. The hybrid strain crossed from the DBA/2 and CBA mice showed little susceptibility to C. neoformans. Thus, this observation makes the usefulness of the hybrid mice for immunological studies doubtful. neoformans passively inhibits phagocytosis of the yeast by macrophages. J Immunol 1982; 129: 1675-1680.

3. Salkowski CA, Bartizal KF, Balish MJ. Balish E. Colonization and pathogenesis of Cryptococcus neoformans in gnotobiotic mice. Infect Immun 1987; 55: 2000-2005. 
4. Baes H, Van Cutsem J. Primary cutaneous cryptococcosis Dermatologica $1985 ; 171$ : 357-361.

5. Rinaldi MG, Drutz DJ, Howell A, Sande MA, Wofsy CB, Hadley WK. Serotypes of Cryptococcus neoformans in patients with AIDS. $J$ Infect Dis 1986; 153: 642.

6. Gupta S, Ellis M, Cesario T, Ruhling M, Vayuvegula B. Disseminated cryptococcal infection in a patient with hypogammaglobulinemia and normal T cell functions. $\mathrm{Am}$ J Med 1987; 82: 129--131.

7. Abrahams I. Further studies on acquired resistance to murine cryptococcosis: enhancing effect of Bordetella pertussis. $J$ Immunol 1966; 96: 525-529.

8. Dykstra MA, Friedman L. Pathogenesis, lethality and immunizing effect of experimental cutaneous cryptococcosis. Infect Immun 1978; 20: 446-455.

9. Graybill JR, Hague M, Drutz DJ. Passive immunization in murine cryptococcosis. Sabouraudia 1981; 19: 237-244.

10. Dromer F, Charriere J, Contrepois A, Carbon C, Yeni P. Protection of mice against experimental Cryptococcus infection by anti-Cryptococcus neoformans monoclonal antibodies. Infect Immun 1987; 55: 749-752.

11. Shibuya K, Naoe S, Uchida K, Yamaguchi H. Histopathological study of murine pulmonary cryptococcosis. Jpn J Med Mycol 1988; 29: 182-190.

12. Van Cutsem J, Fransen J, Van Gerven F, Janssen PAJ. Experimental cryptococcosis: dissemination of Cryptococcus neoformans and dermotropism in guinea-pigs. Mykosen 1986; 29: 561-575.

13. Dixon DM, Polak A. In vivo and in vitro studies with an atypical rhinotropic isolate of Cryptococcus neoformans. Mycopathologia 1986; 96: 33-40.

14. Bergman F. Pathology of experimental cryptococcosis. A study of course and tissue response in subcutaneously induced infection in mice. Acta Pathol Microbiol Scand 1961; Suppl 147: $1-163$.

15. Love GL, Boyd GA, Greer DL. Large Cryptococcus neoformans isolated from brain abscess. J Clin Microbiol 1985; 22 : 1068-1070.

16. Kwong-Chung KJ, Polacheck I, Bennett JE. Improved diagnostic medium for separation of Cryptococcus neoformans var neoformans (serotypes $\mathrm{A}$ and $\mathrm{D}$ ) and Cryptococcus neoformans var gatti (serotypes B and C). J Clin Microbiol 1982; 15: 535-537.

17. Jungerman PF, Schwartzman RM. Cryptococcosis. In: Veterinary medical mycology. Philadelphia, Lea and Feibiger. 1972: 151 .

18. Casadevall A, Mukherjee J, Devi SJN, Schneerson R, Robbins JB, Scharff MD. Antibodies elicited by a Cryptococcus neoformans - tetanus toxoid conjugate vaccine have the same specificity as those elicited in infection. $J$ Infect Dis 1992; 165: 1086-1093.

19. Song MM. Experimental cryptococcosis of the skin. Sabouraudia 1974; 12: 133-137.

20. Seibold HR, Roberts CS, Jordan EM. Cryptococcosis in a dog. $J$ Am Vet Med Ass 1953; 122: 213-115.

21. Kwon-Chung KJ, Bennett JE. Epidemiologic differences between the two varieties of Cryptococcus neoformans. Am J Epidemiol 1984; 120: 123-130. 